\title{
Zika virus outbreak: an overview of the experimental therapeutics and treatment
}

\author{
Shailendra K. Saxena ${ }^{1}\left(\right.$ Asif $^{-}$Elahi $^{1} \cdot$ Srinivasulu Gadugu $^{1} \cdot$ Anil K. Prasad $^{1}$
}

Received: 5 February 2016/ Accepted: 10 February 2016/Published online: 20 February 2016

(C) Indian Virological Society 2016

\begin{abstract}
Zika virus (ZIKV) infection is a new emerging threat around the globe which might be responsible for microcephaly and Guillain-Barre syndrome in the infants. Recently, ZIKV outbreak has caused a public health crisis in Brazil after being linked to a sharp increase in birth defects. ZIKV is ssRNA virus belongs to the family Flaviviridae. It is mainly transmitted by mosquito bite specifically Aedes species and disease symptoms include fever, joint pain, muscle pain, rash, conjunctivitis, and headache. The reservoir of ZIKV is still not known. Protection at personal level by avoiding mosquito bite would help to reduce the incidence of the disease. Control of ZIKV infection by vaccination or antiviral drug either from modern, complementary and alternative medicines may be considered to be one of the most effective strategies in the long run. Large scale immunization of susceptible human population is highly required to prevent this deadly disease. Attempts should be made as soon as possible to develop effective vaccines or antiviral to prevent ZIKV infection. This article provides a current overview of the experimental therapeutics and treatment options based on modern, complementary and alternative medicines.
\end{abstract}

Keywords Zika virus - Aedes species - Microcephaly · Infectious disease $\cdot$ Treatment $\cdot$ Risk assessment and prevention

Shailendra K. Saxena

shailen@ccmb.res.in; shailen1@gmail.com

1 CSIR-Centre for Cellular and Molecular Biology (CCMB), Uppal Road, Hyderabad, TS 500007, India

\section{Introduction}

Emergence and re-emergence of infectious disease is a major concern worldwide for keeping the people healthy and safe around the globe. Currently, Zika virus (ZIKV) infection is a new threat worldwide, causing serious health issue everywhere and researchers believe that this might be the reason of microcephaly and autoimmune disorder in the infants. ZIKV is ssRNA Flavivirus categorized under Flaviviridae family. ZIKV infection is a mosquito transmitted viral infection, which was isolated in 1947, from the blood of febrile sentinel rhesus monkey in the Zika forest of Uganda [8]. For the first time ZIKV was isolated from Aedes africanus species of mosquito in 1948 from the Zika forest. Later on, a serological survey was conducted at the same time in the nearby regions of Uganda and the residents of those regions showed ZIKV specific antibodies in their blood [7, 8]. In the next two decades, a number of isolates of ZIKV were taken from Aedes africanus in Africa while Aedes aegypti in Malaysia which suggested that these Aedes species are the vectors of ZIKV epidemic [11, 13, 21]. During routine arbovirus surveillance studies, many ZIKV isolates were collected from humans in 1960 and 1970s from West and East Africa [10, 15, 19]. ZIKV was also detected in various parts of Africa and South East Asia in 1950 and 1960s [13]. These statistics confirmed the worldwide presence of ZIKV, spreading from Africa to Southeast Asia and America. Further, ZIKV infection was confirmed during an acute fever study in Indonesia in 1977 [16]. The clinical characteristics of ZIKV infection include rash, fever, headache, dizziness, malaise, anorexia and stomach ache $[2,10,16,19]$. Another outbreak of ZIKV infection came into light in 2007 in Yap State, Federated States of Micronesia. 
Recently, first case of ZIKV infection was reported in Brazil in May 2015. Subsequently, outbreaks were reported throughout the country with high number of congenital malformations including 3500 cases of microcephaly [9]. After 9 months of arrival of ZIKV, microcephaly cases in newborns has been tremendously increased in South America. Additionally, the number of infected patients is increasing as the time moving on; hence, there is an urgent need to find the remedy for the eradication of ZIKV infection for maintaining the heath of the human beings around the globe.

\section{Overview of cellular process of infection}

In spite of the fact that the mechanism of infection of ZIKV is not studied in detail so far, although, it belongs to the Flaviviridae family which is well studied Flavivirus group. Therefore, the replication cycle may be similar like other Flaviviruses (Fig. 1). However, further research is essential to ascertain the same. Flaviviruses contain three structure proteins; the capsid protein $\mathrm{C}$, pre-membrane protein prM, and the envelope protein E. RNA genome of the virus is encapsulated in multiple copies of the $\mathrm{C}$ protein to frame the viral nucleocapsid. Virus entry into the cell is mediated by the envelope proteins in three relatively discrete steps. The first step involves the attachment of the virus particle to the cell. Second step is the collisions between virus and target cells and third step is the attachment factors which promote infection by increasing the duration of contact between the virus and cell surface. Flaviviruses are internalized via clathrin-mediated endocytosis and after virus entry into the cell, uncoating of the nucleocapsid take place and RNA molecule is translated as a single polyprotein [5]. Amid this procedure, the polyprotein direct its forward and backward translocation over the endoplasmic reticulum (ER) membrane and processed co and post-translationally by cellular and virus-derived proteases into three structural proteins (C, prM, and E) and seven nonstructural (NS) proteins (NS1, NS2A, NS2B, NS3, NS4A, NS4B, and NS5). The $\mathrm{E}$ protein is glycosylated to assure proper folding of the protein [4]. Then, NS proteins initiate replication of the viral genome and newly synthesized RNA is subsequently packaged to form nucleocapsid by the $\mathrm{C}$ protein. The prM and $\mathrm{E}$ proteins form heterodimers oriented into the lumen of the ER. Then, the prM/E

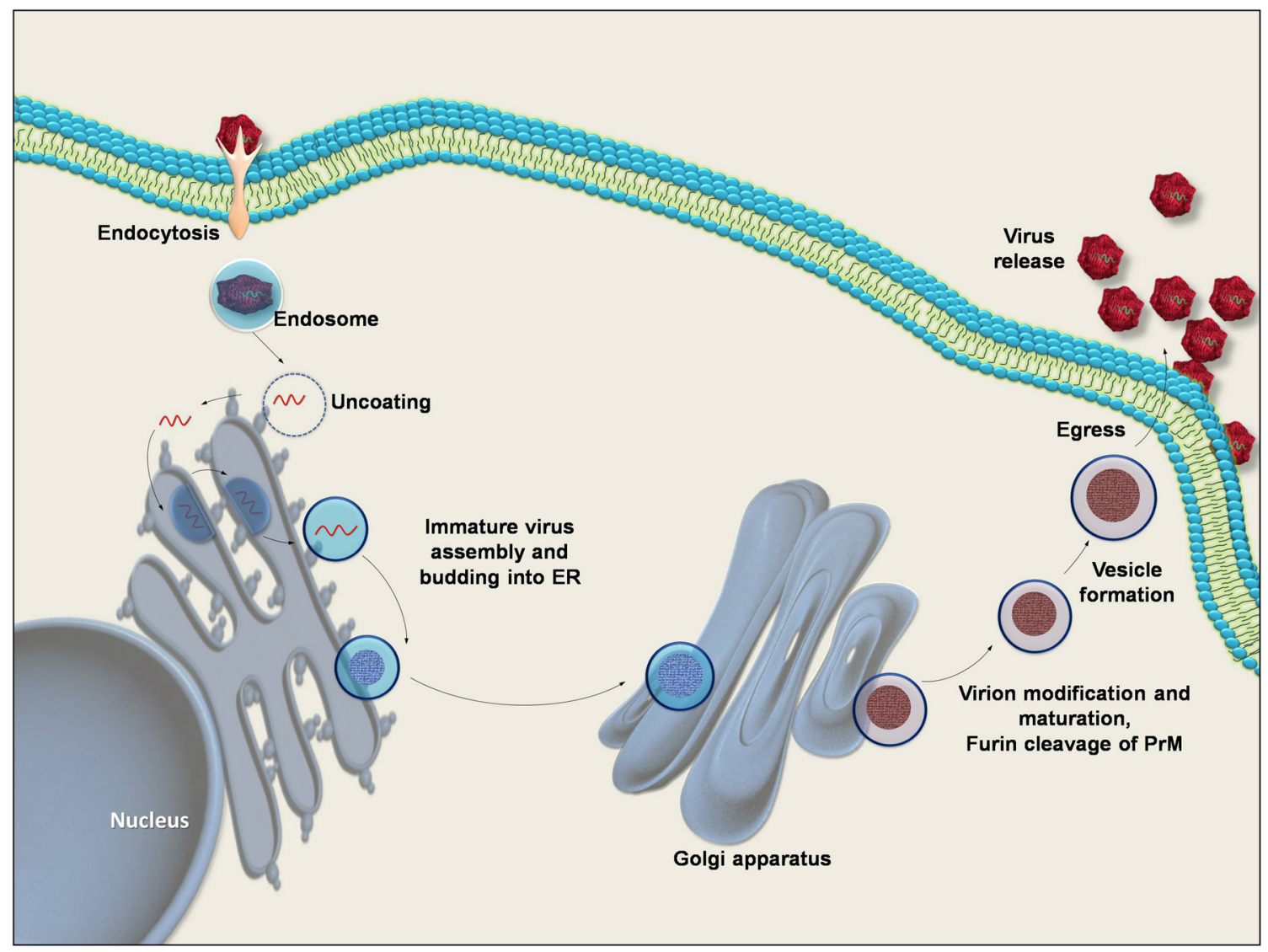

Fig. 1 Infographic exhibiting anticipated Zika virus replication cycle. Zika virus may hijack host cell machinery to facilitate viral replication like other Flaviviruses 
heterodimers associate into trimers and these oligomeric interactions are believed to induce a curved surface lattice, which guides virion budding. Virus particles assemble and bud into the endoplasmic reticulum and are secreted from the cell. During egress, virion maturation occurs in the acidic compartments of the Golgi [18].

\section{Symptoms of ZIKV infection}

The symptoms of ZIKV virus infection are that about 1 in 5 people infected with ZIKV become ill. The clinical presentation includes rash, fever, conjunctivitis and joint pain. Additional symptoms include headache and muscle pain. The incubation period for ZIKV disease is still not clear, but is likely to be 2-7 days with usually mild symptoms of illness. Amid substantial outbreaks in Brazil and French Polynesia in 2015 and 2013 individually, health authorities reported potential auto-immune and neurological entanglements which might be due to ZIKV illness. Recently Brazilian local health authorities have watched an expansion of ZIKV infection in overall population and additionally an increment of microcephaly in infants in northeast Brazil. Offices examining ZIKV episodes are searching an expanding assemblage of confirmation of the association between ZIKV infection and microcephaly. Nonetheless, more examination is required before we appreciate the relationship between microcephaly in babies and the ZIKV infection. Other potential causes are in like manner being explored [22]. Researchers have associated the ZIKV infection with microcephaly, however, the connection between ZIKV and brain abnormalities isn't completely understood, but it may be possible that the virus attacks stem cells during early brain development while also causing the general destruction of other brain tissue. The early loss of stem cells may keep a fetal brain from forming the correct architecture.

\section{Transmission}

ZIKV is transmitted to people through the infected mosquito bite belongs to Aedes family, basically Aedes aegypti in tropical locales. This is the same mosquito that transmits chikungunya, yellow fever and dengue. ZIKV infection episode was represented the first run through from the Pacific in 2007 and 2013 in Yap and French Polynesia, and in 2015 from the Africa (Cape Verde) and Americas (Brazil and Colombia). According to WHO, several nations have reported ZIKV infection in the Americas demonstrating the quick geographic extension [22].

\section{Therapeutics and treatment}

Individuals with Zika infection should get enough water, complete rest, and treat fever and agony with liquid solutions. On the off chance that manifestations exacerbate, they ought to look for therapeutic consideration and counsel. Although, no specific vaccine or medications are available to prevent or treat ZIKV infections so far, but medicine such as acetaminophen or paracetamol may be helpful to relieve fever and pain (Fig. 2). According to US Centre for Disease Control and Prevention (CDC) it is advised not to take nonsteroidal anti-inflammatory drugs (NSAIDs) like aspirin. If individuals are taking medicine for another medical condition, advice of the healthcare provider should be taken before taking additional medication [20].

Based on symptoms, till date there is no particular vaccine has been developed nor advised even though it falls in Flavivirus genus, which is much studied family of viruses. Herbal medicines might be effective therapeutic antimicrobial compounds, considering no or low toxicity. In various countries, these therapeutic herbs are essential source of various intense prescriptions. Medicinal plants contain a number of secondary metabolites, for instance, saponins, flavonoids, alkaloids, and sterols which showed antimicrobial potential. [6]. Alkaloids are heterocyclic ring containing nitrogenous compounds that have therapeutics significance due to their antimicrobial potential and their capacity to bind with the genetic material [12]. All traditional system of medicine specifically homeopathy and ayurveda, may be attempted in ZIKV case, the reason is that homeopathic preparation has already shown effective in case of Japanese encephalitis virus [Saxena and Gadugu. Pers. Comm.,] which falls in the same genus like ZIKV.

\section{Prospective homeopathic medicines for ZIKV}

Homeopathic prescriptions Eupatorium perfoliatum, Atropa belladonna, Rhus tox may be securely utilized as a part of ZIKV treatment. These medications come the nearest in treating the symptoms of ZIKV infection disease. Homeopathy has been very effective in treating epidemic diseases such as cholera, dengue fever, yellow fever typhus, and conjunctivitis. In an epidemic when an immense number of individuals are assaulted by acute and similar sufferings from similar cause, homeopathy may be an excellent prophylactic help. Records demonstrate that during epidemics, homeopathic pharmaceuticals are of great help in diminishing the mortality when contrasted with those put under conventional system of medicines.

Atropa belladonna or Belladonna is a medicinal plant categorized under Solanaceae family. This plant has great commercial importance as it is a major source of 
Fig. 2 Infographic exhibiting recommendations for the treatment of Zika virus infection. The presentation includes an overview of the experimental therapeutics and treatment based on complementary and alternative medicines available options and

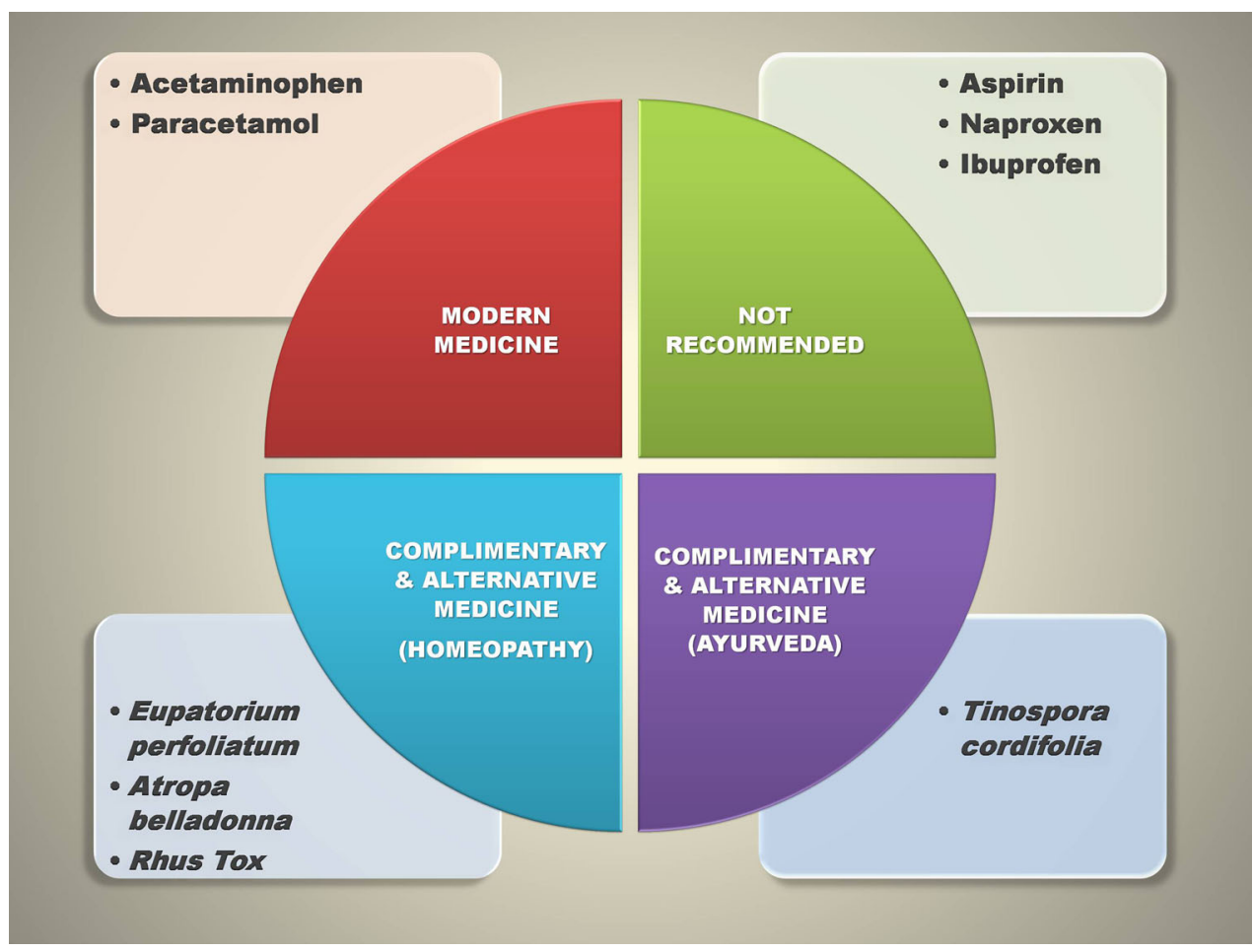

pharmaceutical bioactive compounds like alkaloids mainly scopolamine and hyoscyamine and is native to Western Asia Europe and North Africa. Although, all alkaloids in Atropa belladonna are distributed in the entire plant, but the majority of the alkaloid content is found in green leaves and ripe fruit. It has long been used in human medicine for the treatment of inflammation, headache, peptic ulcer, menstrual symptoms and histaminic reaction [3, 17]. In our studies Belladonna treatment successfully reduced the Japanese encephalitis virus infection [Saxena and Gadugu. Pers. Comm.; [1]]. This may be attempted in case of ZIKV after proper consultation with the physician, since they both belong to same family. In homeopathy, ultra-diluted concentration like 3, 6, 30, and 200 of above mention drugs are prescribed for the treatment of all infectious diseases. Therefore, homeopathy may have a huge role to play as prophylactic when disease spreads suddenly over a substantial region. Additionally, homeopathic pharmaceutical Eupatorium perfoliatum may be utilized as prophylactic as a part of ZIKV, as this drug has the nearest match to the symptoms displayed in ZIKV disease.

\section{Prospective ayurveda medicines for ZIKV}

Ayurveda is an ancient medical science which is based on naturally occurring herbal medicine and showed some effective preventive and healing answers for most of the sicknesses. The most significant aspect of the ayurvedic system of medicine is that, they are entirely based on naturally occurring substances and, therefore, absolutely safe for human consumption without showing any side effect. It has been asserted that Tinospora cordifolia is one of the effective natural solutions for the counteractive action of any sort of viral disease. It is considered as a potential immunomodulator. It is thought that, it reinforces the safe framework and assembles resistance in the body to battle infections. Tinospora Cordifolia is likewise an effective solution for treating fevers particularly from unknown reasons. This herb, which has been utilized as a part of Ayurveda, since hundreds of years, is extremely useful in boosting up immune function and the body's capacity to fight against infections. Ayurvedic herbs helps in enhancing the phagocytic capability of the immune cells especially macrophages. The astringent properties present in the medication show against occasional and antispasmodic properties which are again useful in forestalling infectious sicknesses like intestinal sickness, dengue and swine influenza [14]. Tinospora cordifolia has also shown its effectiveness in case of other diseases like dengue fever, swine influenza and urinary tract infections hence may be an effective remedy for ZIKV infection too.

\section{Prevention}

Mosquitoes and their reproducing locales represent a critical danger component for ZIKV. Preventive measure and control depends on reducing the source of mosquitoes and by decreasing the encounter between humans and mosquitoes. 
This could be possible by utilizing mosquito repellent, using mosquito nets, shutting off screens, entryways and windows. It is likewise critical to look at the places where mosquitoes can breed. Insecticide sprays suggested by the WHO Pesticide Evaluation Scheme might likewise be utilized as larvicides to treat generally extensive water compartments [22]. Rapid test for ZIKV infection should be taken into the consideration during blood transfusion. Before travelling to a new place, a complete record of the diseases endemic to that particular place may also aid in preventing risk. Vector control program should be initiated to prevent the quick spreading of the ZIKV infection. If symptoms of the disease experienced, consultation of certified doctor should be immediately taken Instead of self medication. Women who are planning to conceive the child should require taking extra precautions as microcephaly may be somehow associated with ZIKV infection. Pregnancy may be decisively avoided till the eradication of ZIKV infection.

\section{Future prospective and conclusions}

As the number of cases of ZIKV infection is increasing dramatically, some necessary steps should be taken to eradicate this deadly infection and to inhibit the entry of it in future as well. There is an urgent need of a rapid molecular diagnostics specific for ZIKV so that the infection may be detected in a short period of time and necessary action can be taken before the situation gets out of control. After the development of the diagnostic tools, effective specific therapeutics needs to be developed, for the treatment of ZIKV infection. ZIKV specific vaccine is the need of the hour, which may be developed using modern molecular biology techniques. Apart from the development of diagnostic and therapeutic, researchers and policy makers should come on the same table to discuss the detailed information about the ZIKV outbreak, which may be helpful in the better understanding of the ZIKV infection, prevention and for the development of effective future diagnosis and treatment. We need to proceed with a sense of urgency in this context.

Acknowledgments The authors report no competing financial interest and are responsible for the content and writing of the paper, which is the explicit work of authors. Authors are grateful to the Director, Centre for Cellular and Molecular Biology and Council of Scientific and Industrial Research (CSIR-CCMB), India for the encouragement and support for this work. SK Saxena is also supported by US National Institute of Health Grants: R37DA025576 and R01MH085259.

\section{References}

1. Bandyopadhyay B, Das S, Sengupta M, Saha C, Das KC, Sarkar D, Nayak C. Decreased intensity of Japanese encephalitis virus infection in chick chorioallantoic membrane under influence of ultradiluted Belladonna extract. Am J Infect Dis. 2010;6:24-8.

2. Bearcroft WG. Zika virus infection experimentally induced in a human volunteer. Trans R Soc Trop Med Hyg. 1956;50:442-8.

3. Bousta D, Soulimani R, Jarmouni I, Belon P, Falla J, Froment N, Younos C. Neurotropic, immunological and gastric effects of low doses of Atropa belladonna L., Gelsemium sempervirens L. and Poumon histamine in stressed mice. J Ethnopharmacol. 2001;74:205-15.

4. Bryant JE, Calvert AE, Mesesan K, Crabtree MB, Volpe KE, Silengo S, Kinney RM, Huang CY, Miller BR, Roehrig JT. Glycosylation of the dengue 2 virus E protein at N67 is critical for virus growth in vitro but not for growth in intrathoracicallyinoculated Aedes aegypti mosquitoes. Virology. 2007;366:415-23.

5. Clyde K, Kyle JL, Harris E. Recent advances in deciphering viral and host determinants of dengue virus replication and pathogenesis. J Virol. 2006;80:11418-31.

6. Cowan MM. Plant products as antimicrobial agents. Clin Microbiol Rev. 1999;12:564-82.

7. Dick GW. Zika virus pathogenicity and physical properties. Trans R Soc Trop Med Hyg. 1952;46:521-34.

8. Dick GW, Kitchen SF, Haddow AJ. Zika virus isolations and serological specificity. Trans R Soc Trop Med Hyg. 1952;46:509-20.

9. Dyer O. Zika virus spreads across Americas as concerns mount over birth defects. BMJ. 2015;351:h6983.

10. Fagbami AH. Zika virus infections in Nigeria: virological and seroepidemiological investigations in Oyo State. J Hyg (Lond). 1979;83:213-9.

11. Haddow AJ, Williams MC, Woodall JP, Simpson DIH, Goma LK. Twelve isolations of Zika virus from Aedes (Stegomyia) africanus (Theobald) taken in and above a Ugandan forest. Bull World Health Organ. 1964;31:57-69.

12. Jayasurriya H, Koonchanok MK, Geahlen RL, Mclanghlin JL, Chang CJ. Emodine, a protein kinase inhibitor from polygonum Cuspidatum. J Nat Pro. 1991;55:696-8.

13. Marchette NJ, Garcia R, Rudnick A. Isolation of Zika virus from Aedes aegypti mosquitoes in Malaysia. Am J Trop Med Hyg. 1969;18:411-5.

14. Mittal J, Sharma MM, Batra A. Tinospora cordifolia: a multipurpose medicinal plant- A review. J Med Plant Stud. 2014;2:32-47.

15. Moore DL, Causey OR, Carey DE, Reddy S, Cooke AR, Akinkugbe FM, David-West TS, Kemp GE. Arthropod-borne viral infection of man in Nigeria, 1964-1970. Ann Trop Med Parasitol. 1975;69:49-64.

16. Olson JG, Ksiazek TG. Suhandiman, Triwibowo. Zika virus, a cause of fever in Central Java, Indonesia. Trans R Soc Trop Med Hyg. 1981;75:389-93.

17. Rita P, Animesh DK. An updated overview on Atropa belladonna L. Int Res J Pharm. 2011;2:11-7.

18. Rodenhuis-Zybert IA, Wilschut J, Smit JM. Dengue virus life cycle: viral and host factors modulating infectivity. Cell Mol Life Sci. 2010;67:2773-86.

19. Simpson DIH. Zika virus infection in man. Trans $R$ Soc Trop Med Hyg. 1964;58:335-8.

20. US Centers for Disease Control and Prevention (CDC) (2016) National center for emerging and zoonotic infectious diseases (NCEZID), Division of vector-borne diseases (DVBD). Zika virus. http://www.cdc.gov/zika. Accessed 4 Feb 2016.

21. Weinbren MP, Williams MC. Zika virus: further isolations in the Zika area and some studies on the strains isolated. Trans R Soc Trop Med Hyg. 1958;52:263-8.

22. World Health Organization (2016) Zika virus: Fact sheet (Jan 2016) http://www.who.int/mediacentre/factsheets/zika/en/. Accessed 3 Feb 2016. 